[8] A. B. Simon, Cesàro summability on groups: Characterization and inversion of Fourier transforms, Function Algebras, Proc. International Symposimm, Tulane University, 1965, pp. 208-215.

[9] I. Tweddle, Weak compactness in locally convex spaces, Glasgow Math. J. (1968), pp. 123-127.

[10] J. S. W. Wong, On a characterization of Fourier transforms, Monatshefte fuir Math. 70 (1966), pp. $74-80$.

\section{On relatively disjoint families of measures, with some applications to Banach space theory}

by

HASKELL P. ROSENTHAL* (Berkeley, Calif.)

\section{INTRODUCTION}

We give here details of the results announced in [21], and also extend these results to situations involving sets of arbitrary cardinality. Thus in [21] we proved that every injective Banach space of infinite dimension contains an isomorph of $l^{\infty}$; here we prove that if an injective Banach space contains an isomorph of $c_{0}(T)$ for some set $\Gamma$, it contains an isomorph of $l^{\infty}(T)$ (Corollary 1.5 below). From this we deduce easily the result of Amir [1] that $l^{\infty} / c_{0}$ is not injective, and assuming the continuum hypothesis, that if $K$ is a closed subset of $\beta N$ such that $C(K)$ is injective, then $\Pi$ is Stonian (Corollary 1.6). ( $\beta N$ denotes the Stone-Čech compactification of $N$, the discrete set of positive integers).

These results are consequences of the key Proposition 1.2 which asserts that if $T: l^{\infty}(T) \rightarrow B$ is an operator such that $T \mid c_{0}(T)$ is an isomorphism (i.e. $T \mid c_{0}(\Gamma)$ is one-one with closed range), then there is a $\Gamma^{\prime} \subset \Gamma$ with card $\Gamma^{\prime}=\operatorname{card} \Gamma$ such that $T \mid l^{\infty}\left(\Gamma^{\prime}\right)$ is an isomorphism. (Throughout, "operator" [resp. "projection"] refers to a "bounded linear (Throughout, "operat "bounded linear projection"]. Throughout the introduction, $B$ and $X$ denote Banach spaces and $I$ and $A$ denote infinite sets). Proposition 1.2 in turn yields the considerably stronger Theorem 1.3, (het if $X$ is complemented in $X^{* *}$ and $X$ contains an isomorph of $c_{0}(T)$, then $X$ contains an isomorph of $l^{\infty}(\Gamma)$. (We regard $X$ as being canonically imbedded in $X^{* *}$.) Theorem 1.3 can also be used to prove a result concerning extensions of isomorphisms of subspaces of $l^{\infty}(T)$ into injective Banach spaces, thus generalizing a result in [13]. (Cf. Corollary 1.7 and the Theorem following it.) 
Proposition 1.2 and most of the results of this paper are consequences of a lenma which generalizes Lemma 1 of [21] (which in turn generalizes "Phillip's Lemma" [20]). We state this lemma (Lemma 1.1) in Section 1 and deduce there its consequences for injective Banach spaces mentioned above. Section 2 is devoted to the proof of 1.1 ; the argument is selfcontained, and is a generalization of the proof of Lemma 1 of [21]. In a sense this argument combines the approaches of Phillips [20] and Nakamura and Kakutani [14]. (For applications of the latter, c.f. [11], [19], and [26]).

In Section 3 we introduce the notions of relatively disjoint and strongly relatively disjoint families of measures. (We feel that these notions, already implicit in the literature for some time (e.g., c.f. [7], $[9],[10],[16]$, and [18]), underlie most of the results of this paper. However, Sections 1 and 2 do not explicitly use these concepts). After showing in Proposition 3.1 the essentially known result that infinite relatively disjoint families in $l^{1}(T)$ span complemented subspaces isomorphic to $l^{1}(\Lambda)$ for some $\Lambda$, we deduce further consequences of Lemma 1.1 concerning the spaces $l^{1}(I)$ and $c_{0}(I)$. Thus in Theorem 3.3 (resp. Theorem 3.4) we give rather weak sufficient conditions on a subset of $l^{1}(\Gamma)$ (resp. of any $B$ ) such that it be equivalent to the unit-vectors-basis of $l^{1}(I)$ (resp. of $c_{0}(T)$ ). (The conditions are trivially also necessary.) 3.3 may be used to deduce the known result that every infinite-dimensional complemented subspace of $l^{1}(\Gamma)$ is isomorphic to $l^{1}(\Lambda)$ for some $\Lambda$ (due to Pelczyniski for countable $(T)$ [18] and Köthe for the general case [10]), and it also yields a new proof of our Lemma 1.1 of [22] (c.f. the second corollary following 3.3 below). Theorem 3.4 is easily seen to be equivalent to the assertion that if $T$ is an operator from $c_{0}(\Gamma)$ to $B$ such that $\inf \left\|T_{\{\langle\gamma\}}\right\|>0$, then there exists a $\Gamma^{\prime} \subset \Gamma$ such that $\operatorname{card} \Gamma^{\prime}=\operatorname{card} \Gamma$ and $\stackrel{\gamma_{\epsilon} T}{T} \mid c_{0}\left(\Gamma^{\prime}\right)$ is an isomorphism. This yields some new information also for countable $\Gamma$, generalizing Theorem 5 of $[2] .\left(\chi_{\{\nu\}}(\beta)=1\right.$ if $\gamma=\beta,=0$ otherwise.) We conclude Section 3 with applications of strongly relatively disjoint sequences of measures, deducing the results 1-7 of [21]. The arguments given there are already implicitly contained in [21], and yield now proots of some known results (e.g., of Theorem 1 of [17]).

\section{DEFINITIONS AND NOTATIONS}

We follow [3] and [5] for the most part. Banach spaces are taken over either the real or complex scalars. Let $X$ and $Y$ be Banach spaces. The unit ball of $X,\{x \in X:\|x\| \leqslant 1\}$, is denoted by $S_{X}$. An operator $T$ from $X$ to $Y$ is called an isomorphism if it is one-one with closed range. $X$ and $Y$ are called isomorphic (denoted by $X \approx Y$ ) if there is an isomorphism (i.e., invertible operator) mapping $X$ onto $Y$. If $X \subset Y, X$ is said to be complemented if there is a projection mapping $Y$ onto $X$. A Banach space is said to be injective if it is complemented in every Banach space containing it.

Given a set $\Gamma, l^{\infty}(\Gamma)$ denotes the Banach space of all bounded scalarvalued functions defined on $\Gamma$ under the supremum norm; $c_{0}(T)$ denotes the closed subspace of $l^{\infty}(T)$ consisting of all $f$ such that for any $\varepsilon>0$ there exists a finite subset $F_{\varepsilon}$ of $\Gamma$ with $|f(\gamma)|<\varepsilon$ for all $\gamma \notin F_{\varepsilon}$. If $A$ is a subset of $\Gamma, \chi_{A}$ denotes the characteristic function of $A$ (i.e. $\chi_{A}$ is one on $\Lambda$ and zero off $\Lambda$.) If $f$ is a scalar-valued function defined on $\Gamma, f \mid \Lambda$ denotes the function $\chi_{A} f . l^{1}(\Gamma)$ denotes the space of all scalar-valued $f$ defined on $\Gamma$ such that $\|f\|=\sum_{\gamma \in \Gamma}|f(\gamma)|<\infty$, under the norm $\|\cdot\| \cdot \beta \Gamma$ denotes the Stone-Čech compactification of $\Gamma$ and card $\Gamma$ denotes the cardinality of $\Gamma$.

$c$ denotes the cardinality of the set of real numbers and $\boldsymbol{s}_{0}$ the cardinality of the set of integers. If $\operatorname{card} \Gamma=\mathrm{m}$, we denote $l^{\infty}(\Gamma)$ by $l_{\mathrm{m}}^{\infty}$ and $l^{1}(\Gamma)$ by $l_{\mathrm{m}}^{1}$. In the case where $\Gamma=N$, the set of positive integers, we denote $l^{\infty}(\Gamma), c_{0}(\Gamma)$, and $l^{1}(\Gamma)$ by $l^{\infty}, c_{0}$, and $l^{1}$ respectively. (We assume the notation and standard facts concerning cardinal and ordinal numbers, as exposed in [25].)

If $\Sigma$ is a $\sigma$-algebra of subsets of the set $E$, ca $(E, \Sigma)$ denotes the Banach space of countably additive scalar-valued set functions on $\Sigma$, under total variation norm. If $\mu$ is a countably or finitely additive scalarvalued measure defined on $\Sigma,|\mu|$ denotes the total variation of $\mu$ (as defined in [5]).

Given a compact Hausdorff space $S, C(S)$ denotes the Banach space of scalar-valued continuous functions on $S$, under supremum norm. We denote by $M(S)$ the space of all regular scalar-valued Borel measures on $S$, and identify $C(S)^{*}$ with $M(S)$ by the Riesz representation theorem. (Of course we also regard $M(S) \subset \operatorname{ca}(S, \Sigma)$ where $\Sigma$ denotes the $\sigma$-algebra of Borel subsets of $S$.) A subset of $S$ is called clopen if it is both closed and open. $S$ is said to be $\sigma$-Stonian (resp. an $F$-space) if open $F_{\sigma}$ subsets of $S$ have open closures (resp. if disjoint open $F_{\sigma}^{\prime}$ 's have disjoint closures). More generally, $S$ is said to be m-Stonian if the clopen subsets of $S$ form a base for its open sets, and if every family $\mathscr{F}$ of disjoint clopen subsets of $S$ with card $\mathscr{F} \leqslant m$ is such that $\overline{U F}$ is open. (Thus $\sigma$-Stonian $=\boldsymbol{x}_{0}$ Stonian).

$S$ is called Stonian, or extremely disconnected, if the closure of every open subset of $S$ is open. It is easily seen that $S$ is Stonian if and only if $S$ is $m$-Stonian for all cardinals $m$ and that $\beta \Gamma$ is Stonian for all sets $\Gamma$; it is also known that $C(S)$ is injective for any Stonian $S$ (c.f. [3]). Finally, we say that $S$ satisfies the Countable Chain Condition (the C.C.C.) if every family of disjoint open subsets of $S$ is finite or countable. 


\section{SECTION I}

All of our results are consequences of the following lemma whose countable case generalizes a lemma of Phillips (see the last result of this section):

LEMMA 1.1. Let $\Lambda$ be a discrete set and let $\left\{\mu_{\alpha}: \alpha \in \Gamma\right\}$ be an infinite family of finitely additive positive measures on $\Lambda$ such that $\sup _{a \in T} \mu_{\alpha}(\Lambda)<\infty$, and let $\left\{E_{a}: \alpha \in \Gamma\right\}$ be a family of disjoint subsets of $\Lambda$. Then for all $\varepsilon>0$, there exists a $\Gamma^{\prime} \subset \Gamma$ with card $\Gamma^{\prime}=\operatorname{card} \Gamma$ such that

$$
\mu_{a}\left(\bigcup\left\{E_{\beta}: \beta \epsilon \Gamma^{\prime}, \beta \neq \alpha\right\}\right)<\varepsilon \text { for all } \alpha \in \Gamma^{\prime \prime} .
$$

This lemma has some non-trivial consequences also for families of countably additive measures defined on all subsets of $\Lambda$ (i.e., for families contained in $l^{1}(\Lambda)$ ) which we explore in Section 3. We develop its consequences for injective spaces and their quotients in the present section, and delay its proof until Section 2, where we prove the apparently stronger

LEMMA 1.1 (a). Let $\mathrm{m}$ be an infinite cardinal number. Let $S$ be an. m-Stonian compact Hausdorff space, let $\Gamma$ be a set with card $\Gamma=1 \mathrm{t}$, and let $\left\{\mu_{a}: \alpha \in \Gamma\right\}$ and $\left\{E_{\alpha}: \alpha \in \Gamma\right\}$ be given, where for all $\alpha \in \Gamma, \mu_{a}$ is a non-negative member of $M(S)$ with $\left\|\mu_{\alpha}\right\| \leqslant 1$ and $E_{\alpha}$ is a clopen subset of $S$ such that for all $\beta \in \Gamma$ with $\beta \neq \alpha, E_{\alpha} \cap E_{\beta}=\varnothing$. Then given $\varepsilon>0$, there exists a subset $\Gamma^{\prime}$ of $\Gamma$ with card $\Gamma^{\prime}=$ card $\Gamma$, such that for all $\alpha \in \Gamma^{\prime}$,

$$
\mu_{a} \overline{\left(\bigcup\left\{E_{\beta}: \beta \epsilon \Gamma^{\prime}, \beta \neq \alpha\right\}\right)}<\varepsilon .
$$

(Of course if $\mathbb{S}$ is Stonian, this holds for all infinite sets $\Gamma$, with no restriction on $\operatorname{card} \Gamma$.)

Remarks. 1. In view of the fact that to every finitely-additive positive measure $\mu$ on the discrete set $\Lambda$, there corresponds a unique positive $\tilde{\mu} \in M(\beta \Lambda)$ such that $\tilde{\mu}(\bar{E})=\mu(E)$ for all $E \subset \Lambda, 1.1$ is an immediate consequence of 1.1 (a). Actually, following an observation of Grothendieck [7], 1.1 (a) is also a consequence of 1.1. Indeed, let $S, \mathrm{~m}, \Gamma$, $\left\{\mu_{a}: \alpha \in \Gamma\right\}$ and $\left\{E_{a}: a \in \Gamma\right\}$ satisfy the hypothesis of 1.1 (a). For each $\alpha \in \Gamma$, define the set function $v_{a}$ on the discrete set $\Gamma$ by

$$
\nu_{a}(F)=\mu_{a}\left(\bigcup_{a \in F} E_{a}\right) \quad \text { for all } F \subset \Gamma \text {. }
$$

Since $S$ is m-Stonian, it follows that $\nu_{a}$ is finitely additive with $\left\|\nu_{a}\right\| \leqslant 1$; applying Lemma 1.1 to $\left\{\nu_{a}: \alpha \in \Gamma\right\}$ and $\{\{\alpha\}: a \in \Gamma\}$ (the family of singletons of $\Gamma$ ), we obtain for all $\varepsilon>0$, a $\Gamma^{\prime} \subset \Gamma$ with $\operatorname{card} \Gamma^{\prime}=\operatorname{card} \Gamma$ such that $v_{a}\left(\Gamma^{\prime} \sim\{\alpha\}\right)<\varepsilon$ for all $\alpha \in \Gamma^{\prime}$, whence 1.1(a) follows.

2. It is easily seen, using a result of Grothendieck, that 1.1(a) for $m=\boldsymbol{N}_{0}$ is equivalent to Lemma 1 of [21] (see the last result of Section 3).
Our next result provides the key tool for applying 1.1 to injective Banach spaces.

Proposition 1.2. Let $B$ be a Banach space, $\Gamma$ an infinite set, and $T: l^{\infty}(T) \rightarrow B$ an operator such that $T \mid c_{0}(T)$ is an isomorphism. Then there exists $a$ set $\Gamma^{\prime} \subset \Gamma$ with card $\Gamma^{\prime}=\operatorname{card} \Gamma$ such that $T \mid l^{\circ}\left(\Gamma^{\prime}\right)$ is an isomorphism.

Proof. Put $K=\left\|\left(T \mid e_{0}(T)\right)^{-1}\right\|$. Now fix $\gamma \in \Gamma$; then by the HahnBanach Theorem, there exists an $f_{\gamma} \in B^{*},\left\|f_{\gamma}\right\| \leqslant K$, such that

$$
f_{\gamma}\left(T_{\chi_{\{\gamma\}}}\right)=1 \quad\left(\text { since }\left\|T_{\chi_{\{\gamma\}}}\right\| \geqslant 1 / K\right) .
$$

Defining the set function $\mu_{\gamma}$ by $\mu_{\gamma}(E)=T^{*} f_{\gamma}\left(\chi_{E}\right)$ for all $E \subset \Gamma$, we have as is well-known [5] that $\mu_{\gamma}$ is finitely-additive with $\left\|\mu_{\gamma}\right\|=\left\|T^{*} f_{\gamma}\right\|$, and of course $\sup _{\gamma \in T}\left\|\mu_{\gamma}\right\| \leqslant\|T\| K$. Now letting $E_{\gamma}=\{\gamma\}$, we have by Lemma 1.1 that there exists a set $\Gamma^{\prime} \subset \Gamma$ with $\operatorname{card} \Gamma^{\prime}=\operatorname{card} \Gamma$ such that $\left|\mu_{\alpha}\right|\left(\Gamma^{\prime} \sim\{\alpha\}\right)$ $<\frac{1}{2}$ for all $\alpha \in \Gamma^{\prime}$. But then if $\varphi \in l^{\infty}\left(\Gamma^{\prime}\right)$ and if $\alpha \in \Gamma^{\prime}$,

$$
\left|\int \varphi d \mu_{\nu}\right|=\left|\varphi(\alpha)+\int_{\Gamma^{\prime} \sim\{\alpha\}} \varphi d \mu_{\gamma}\right| \geqslant|\varphi(\alpha)|-\frac{1}{2}\|\varphi\|_{\infty}
$$

since $\mu_{a}(\alpha)=T^{*} f_{a}\left(\chi_{a}\right)=1$

Thus

$$
\left\|T_{\varphi}\right\| \geqslant \frac{1}{K} \sup _{\gamma \in \Gamma^{\prime}}\left|f_{\gamma}(T \varphi)\right| \geqslant \frac{1}{2 K}\|\varphi\|_{\infty}
$$

whence $T \mid l^{\infty}\left(\Gamma^{\prime}\right)$ is an isomorphism. Q. E. D.

Remarks. 1. The above argument shows that the conclusion of 1.2 holds if we replace the assumption that $T \mid c_{0}(\Gamma)$ is an isomorphism by the assumption that $\inf _{\gamma \in \Gamma}\left\|T \chi_{\{\gamma\}}\right\|>0$. In this connection, see also Theorem 3.4 below.

2. If $\Gamma$ is countable, 1.2 may be proved without the aid of Lemma 1.1. We identify $l^{\infty}(\Gamma)$ with $C(\beta \Gamma)$, and choose a constant $K$ such that for each $\gamma \in \Gamma$, we can choose an $f_{\gamma} \epsilon B^{*}$ with $\left\|f_{\gamma}\right\| \leqslant K$ and $f_{\gamma}\left(T_{\chi_{\{\beta\}}}\right)=\chi_{\{\gamma\}}(\beta)$ for all $\beta$. (This is possible since $T \mid c_{0}(T)$ is an isomorphism.) Now $\mu_{\gamma}$ is defined as in the proof of 1.2 ; then since $\beta \Gamma \sim \Gamma$ does not satisfy the C.C.C, there exists an infinite subset $\Gamma^{\prime}$ of $\Gamma$ such that $\left|\mu_{a}\right|\left(\bar{\Gamma}^{\prime} \sim \Gamma\right)=0$ for all $a \in I$ (c.f. the footnote following 1.5 below). Then identifying $l^{\infty}\left(\Gamma^{\prime}\right)$ with all $\varphi \epsilon C(\beta \Gamma)$ such that $\varphi$ is supported on $\overline{\Gamma^{\prime}}$ and taking into account that $\mu_{\gamma}(\{\beta\})=\chi_{\gamma}(\beta)$ for all $\beta \in \Gamma$, we have that for such $\varphi, \int \varphi d \mu_{\gamma}=\varphi(\gamma)$ for all $\gamma \in \Gamma^{\prime}$. Hence $T \mid l^{\infty}\left(\Gamma^{\prime}\right)$ an isomorphism since $\|T \varphi\| \geqslant \frac{1}{K}\|\varphi\|_{\infty}$ for all $\varphi \in l^{\infty}\left(\Gamma^{\prime}\right)$. 
We now deduce our next result (the main theorem of this section) from 1.2 .

THEOREM 1.3. Let $\Gamma$ be an infinite set, and let $B$ and $X$ be Banach spaces with $X$ complemented in $X^{* *}$. Let $T: X \rightarrow B$ be an operator sueh that there exists a subspace $A$ of $X$, isomorphic to $c_{0}(T)$, with $T \mid A$ an isomorphism. Then there exists a subspace $Y$ of $X$, isomorphic to $l^{\infty}(T)$, with $T \mid Y$ an isomorphism.

Proof. We first observe that there exists an operator $S^{S}: l^{\infty}\left(I^{\top}\right) \rightarrow X$ such that $S \mid c_{0}(T)$ is an isomorphism onto $A$. Indeed, we identify $l^{\infty}\left(I^{\prime}\right)$ with $\left(c_{0}(I)\right)^{* *}$ and regard as always $X \subset X^{* *}$. Then choose $S_{1}: \quad c_{0}(I) \rightarrow X$ an isomorphism of $c_{0}\left(T^{T}\right)$ with $A$. Then $S_{1}^{* *}: l^{\infty}(I) \rightarrow X^{* *}$ is such that $S_{1}^{* *} \mid c_{0}(T)=S$.

Now let $P$ be a projection from $X^{* *}$ onto $X$; then $S=P S_{1}^{* *}$ is the desired operator.

Thus $T S$ is an operator from $l^{\infty}(T)$ into $B$, such that $T S \mid c_{0}(T)$ is an isomorphism. By Proposition 1.2, we may choose $\Gamma^{\prime} \subset T$ with card $\Gamma^{\prime}$ $=\operatorname{card} \Gamma$, such that $T S \mid l^{\infty}\left(\Gamma^{\prime}\right)$ is an isomorphism. Thus $S \mid l^{\infty}\left(\Gamma^{\prime}\right)$ and $T \mid S\left(l^{\infty}\left(\Gamma^{\prime}\right)\right)$ are both isomorphisms; so putting $Y=S\left(l^{\infty}\left(T^{\prime}\right)\right)$, the result follows. Q.E.D.

Corollary 1.4. Let $X$ and $B$ be Banach spaces and let $T$ be a nonweakly compact operator from $X$ to $B$. Then if $X$ is injective, there exists a subspace $Y$ of $X$ isomorphic to $l^{\infty}$, such that $T \mid Y$ is an isomorphism.

Proof. Since $X$ is injective, there exists a $C(K)$ space $Z$ and a surjective operator $S: Z \rightarrow X$. Then $T S$ is not weakly compact, hence by a result of Pelczyński (Theorem 1 of [17]), there exists a subspace $A_{1}$ of $Z$ with $A_{1} \approx c_{0}$ and $S \mid A_{1}$ an isomorphism. (We give an independent proof of this result of Pelczynskik's in Theorem 3.7 below.) Thus $A=S\left(A_{1}\right)$ is isomorphic to $c_{0}$ and $T \mid A$ is an isomorphism, whence since $X$ is complemented in $X^{* *}$, the result follows from 1.3. Q.E.D.

Remarks. 1. Corollary 1.4 implies, of course, that every injective Banach space contains a subspace isomorphic to $l^{\infty}$ (Corollary 3 of [21]).

2. The above argument easily yields that the conclusion of 1.4 holds provided we assume that $X$ is a continuous linear image of some injective Banach space, or assuming the continuum hypothesis and a result of Tindenstrauss [12], provided $X$ is a continuous linear image of $O(K)$ for some compact $F$-space $K$. It follows from our Theorem 3.7 below that the conclusion also holds without the assumption of the continuum hypothesis, provided $X$ is a continuous linear image of $C(S)$ for some $\sigma$-Stonian $S$.

It is easily seen (and is well-known) that $X$ is complemented in $X^{* *}$ if and only if $X$ is isomorphic to a complemented subspace of some conjugate Banach space. We thus obtain immediately from 1.3.
CoRollary 1.5. Let $X$ be injective, or more generally, let $X$ be isomorphic to a complemented subspace of some conjugate Banach space, and let $\Gamma$ be an infinite set. If $X$ contains a subspace isomorphic to $c_{0}(T)$, then $X$ contains a subspace isomorphic to $l^{\infty}(T)$.

Remarks. 1. In [22] we proved that if $X$ is a $\mathscr{P}_{1}$ space (i.e. $X=C(S)$ for some Stonian $S$ ) containing a subspace isomorphic to $c_{0}(I)$, then $X$ contains a subspace isometric to $l^{\infty}(T)$. We also proved in Corollary 1.2 of [22] that if $B$ is a Banach space such that $B^{*}$ contains an isomorph of $c_{0}(T)$, then $B$ contains a complemented isomorph of $l^{1}(T)$ and hence $B^{*}$ contains an isomorph of $l^{\infty}(\Gamma)$. This result is due to Bessaga and Pelczyński for the case of countable $\Gamma$ [2]. (The main tool of proof of 1.2 of [22] was Lemma 1.1 of [22]; we give a new proof of 1.1 of [22] using our present Lemma 1.1 in Section 3 (c.f. the second Corollary following Theorem 3.3 below).)

2. Let $X$ be a complemented subspace of $l^{\infty}(T)$, containing an isomorph of $c_{0}(T)$. Then by 1.5, $X$ contains an isomorph of $l^{\infty}(T)$. Since $l^{\infty}(T)$ is isomorphic to $\left(l^{\infty}(\Gamma) \oplus l^{\infty}(\Gamma) \oplus \ldots\right)_{\infty}$, we obtain by the "decomposition method" of Pełczyński [18] that $X$ and $l^{\infty}(\Gamma)$ are isomorphic (c.f. Proposition 1.4 of [22]). This result is due to Lindenstrauss (unpublished for uncountable $\Gamma$; proved in [11] for countable $\Gamma$ ).

We obtain immediately from 1.5 and known results the following result of Amir:

COROLLART (Amir [1]). $l^{\infty} / c_{0}$ is not an injective Banach space.

Proof. It is well-known that $l^{\infty} / c_{0}$ contains a subspace isometric to $e_{0}(\Gamma)$ where $\operatorname{card} \Gamma=\mathrm{c}$. $\left.{ }^{1}\right)$ But since card $l^{\infty} / c_{0}=c$, it is impossible that $l^{\infty} / c_{0}$ contain a subspace isomorphic to $l^{\infty}(\Gamma)$, for $\operatorname{card} l^{\infty}(\Gamma)=2^{\text {c }}$. Thus the result follows by Corollary 1.5. Q.E.D.

The preceding result shows that $C(\beta N \sim N)$ is not injective. If we assume a hypothesis weaker than the continuum hypothesis, we obtain a result which implies that if $S$ is a closed subset of $\beta N$ such that $C(S)$ is injective, then $S$ is extremely disconnected.

We first need the simple

Proposition. Let $K$ be a compact F-space satisfying the Countable Chain Condition; then $K$ is Stonian.

Proof. Let $U$ and $V$ be disjoint open subsets of $K$. By Zorn's lemma, we may choose maximal families $\mathscr{F}$ and $\mathscr{G}$ of pairwise disjoint open $F_{\sigma}$

(1) Identify $l^{\infty}$ with $l^{\infty}(Q)$ where $Q$ is the set of rational numbers. For each real number $r$, choose a sequence $\left(x_{n}^{r}\right)$ of distinct rationals tending to $r$, and put $F_{r}=\left\{x_{n}^{r}: n=1,2, \ldots\right\}$. Then $F_{r} \cap F_{r}$ is finite if $r \neq r^{\prime}$. Let $\pi: l^{\infty} \rightarrow l^{\infty} / c_{0}$ be the quotient map; then the closed linear span of $\left\{\pi \%_{F_{r}}: r\right.$ is a real number $\}$ is isometric to $c_{0}(\Gamma)$ where $\operatorname{card} \Gamma=\mathrm{c}$. 
subsets of $U$ and $V$ respectively. Then by maximality, $\overline{U F}=\bar{U}$ and $\overline{U \mathscr{G}}=\bar{\nabla}$. Since $K$ satisfies the c.c.c., $\mathscr{F}$ and $\mathscr{G}$ are countable, thus $\cup \mathscr{F}$ and $\cup \mathscr{G}$ are disjoint open $F_{\sigma}$ sets, so $\bar{U} \cap \bar{V}=\emptyset$. Q.E.D.

Remark. It follows that if $\mu \in M(K)$ and $K$ is an $F$-space, then the support of $\mu$ is Stonian, since the support of $\mu$ is an $F^{3}$-space satisfying the C.c.0. This result is due to Seever [24].

Corollary 1.6. Assume the 7ypothesis $2^{\mathrm{K}_{0}}<2^{\mathrm{\aleph}_{1}}$. Let S be a compact $F$-space with weight $S=\mathfrak{c}$, such that $C\left(S^{\prime}\right)$ is injective. Then $S$ is Stonian. (The weight of $S$ is defined as the smallest cardinal corresponding tio a base for the open sets of $S$.)

Proof. By the Proposition, it suffices to prove that $S$ satisfies the Countable Chain Condition. Suppose that $\&$ does not satisfy the C.C.C. Then there exists a family $T$ of pairwise disjoint open subsets of $S$, with $\operatorname{card} \Gamma=\aleph_{1}$, and hence $C(S)$ contains a subspace isometric to $c_{0}\left(I^{\prime}\right)$ (choose for each $\gamma \in \Gamma$ a non-zero $\varphi_{\gamma} \in O(S)$ supported in $\gamma$, and then take the closed linear span of $\left.\left\{\varphi_{\gamma}: \gamma \in \Gamma\right\}\right)$. Thus by Corollary 1.5 and our hypotheses, $C(S)$ contains a subspace isomorphic to $l^{\infty}(T)$. Since card $l^{\infty}(\Gamma)=2^{\mathrm{N}_{1}}$, card $C(S) \geqslant 2^{\mathrm{K}_{1}}$. But weight $S=\mathfrak{c} \Rightarrow \operatorname{card} C(S)=\mathfrak{c}=2^{\mathrm{N}_{0}}<2^{\mathrm{K}_{1}}$, a contradiction. Q.E.D.

Questron: Does there exist a compact $F$-space $S$ such that $G(S)$ is injective yet $S$ is not Stonian?

We know only that the answer is affirmative if there exists a measurable infinite cardinal $\mathrm{m}$. ( $\mathrm{m}$ is said to be measurable, if letting $l^{\prime}$ be a set with $\operatorname{car} d \Gamma=\mathrm{m}$, there is a non-zero countably-additive measure $\mu$ defined on all subsets of $T$, taking values either 0 or 1 , such that $\mu\{\gamma\}=0$ for all $\gamma \in \Gamma$.)

Our next result may be used to generalize one of the results of [13] concerning extensions of certain isomorphism; it is a simple consequence of Theorem 1.3. (If $A$ and $B$ are closed subspaces of the Banach space $X$, then we write $A \perp B$ ( $A$ is perpendicular to $B$ ) provided $A \cap B=\{0\}$ and $A+B$ is closed.)

Corollary 1.7. Let $X$ be an injective Banach space, $A$ a closed subspace of $X$, and $\Gamma$ an infinite set. Suppose there exists a closed subspace $B$ of $X$, isomorphic to $c_{0}(T)$, such that $A \perp B$. Then there exists a projection $P$ from $X$ onto a subspace isomorphic to $l^{\infty}(\Gamma)$, such that $P A=\{0\}$.

Proof. Let $\pi: X \rightarrow X / A$ be the quotient map. Then $\pi \mid B$ is an isomorphism. Hence by Theorem 1.3, there exists a subspace $D$ of $X$ with $D \approx l^{\infty}(T)$ such that $\pi \mid D$ is an isomorphism. Since $l^{\infty}(T)$ is injective, there exists a projection $Q$ from $X / A$ onto $\pi(D)$. Then $P=(\pi \mid D)^{-1} Q \pi$ is the desired projection. Q.E.D.

Now an argument similar to the proof of Theorem 3 of [13] yields the following
Theorem. Let $X$ be an injective Banach space, $Y$ a closed subspace of $l^{\circ}(\Gamma)$ for some set $T$, and $T: Y \rightarrow X$ an isomorphism. Suppose that there exists $a B \subset X$ with $B \approx c_{0}(T)$ and $B \perp T(Y)$. Then there exists an isomorphism $\tilde{T}: l^{\infty}(T) \rightarrow X$ cxtending $T$ (i.e. such that $\tilde{T} \mid Y=T$ ).

Remark. As was observed in [13], such a $B$ always exists if $\Gamma$ is countable and $X / T(Y)$ is non-reflexive (this also follows immediately from our Theorem 3.7 below). If $X=l^{\infty}(T)$ itself then it follows from our Proposition 3.5 below that such a $B$ exists if $\operatorname{dim} Y<\operatorname{dim} l^{\infty}(\Gamma)$ (in fact if $Y$ contains no isomorph of $l_{2}^{1} \mathrm{mt}$ where $\mathrm{m}=\operatorname{card} \Gamma$; see the remark following 3.5).

We conclude this section by showing that Phillip's Lemma is a rather easy consequence of Lemma 1.1 for the case of countable $\Gamma$ (i.e. of Lemma 1 of [21]).

THEOREM. Let $\left(\mu_{n}\right)$ be a sequence of finitely additive set functions (complex valued) defined on the discrete set $\Lambda$.

(a) (Dieudonné [4]) If $\sup _{n}\left|\mu_{n}(E)\right|<\infty$ for all $E \subset A$, then

$$
\sup _{n}\left\|\mu_{n}\right\|<\infty \text {. }
$$

(b) (Phillips [20]) If $\lim _{n} \mu_{n}(E)=0$ for all $E \subset A$, then

$$
\lim _{n} \sum_{j \in, 1}\left|\mu_{n}(j)\right|=0 .
$$

Proof. Suppose first that $\left(\mu_{n}\right)$ satisfies the hypotheses of (a), yet $\sup _{n}\left\|\mu_{n}\right\|=\infty$. Put $\lambda(E)=\sup _{n}\left|\mu_{n}(E)\right|$ for all $E \subset \Lambda$, and choose $\nu_{1}, v_{2}, \ldots$ a subsequence of the $\mu_{n}$ 's and $E_{1}, E_{2}, \ldots$ subsets of $A$ such that

$$
\left|v_{n}\left(E_{n}\right)\right| \geqslant\left\|r_{n}\right\| / 5 \geqslant n+2 \sum_{j=1}^{n-1} \lambda\left(E_{j}\right) \quad \text { for all } n>1 .
$$

Then putting $F_{n}=E_{n} \sim \bigcup_{j=1}^{n-1} E_{j}$ for all $n>1$ (and $F_{1}=E_{1}$ ), we have that for all $n,\left|v_{n}\left(F_{n}\right)\right| \geqslant\left\|v_{n}\right\| / 10$ and $F_{n} \cap F_{m}=\varnothing$ for all $n \neq m$. By Lemma 1.1, we may choose $n_{1}<n_{2}<\ldots$ such that for all $i$,

$$
\left|v_{n_{i}}\right|\left(\bigcup_{j \neq i} F_{n_{j}}\right)<\frac{1}{2}\left|v_{n_{i}}\left(F_{n_{i}}\right)\right| .
$$

(We apply 1.1 to the measures $\left|v_{n}\right| /\left|v_{n}\left(F_{n}\right)\right|$ and put $\varepsilon=\frac{1}{2} ;\left|v_{n}\right|(\Lambda) /\left|v_{n}\left(F_{n}\right)\right|$ $<10$ for all $n$, of course.) Then putting $F=\bigcup_{j=1}^{\infty} F_{n_{j}}$,

$$
\left|v_{n_{i}}\left(F^{\prime}\right)\right| \geqslant\left\|v_{n_{i}}\right\| / 10, \quad \text { whence }\left|v_{n_{i}}\left(F^{\prime}\right)\right| \rightarrow \infty,
$$

a contradiction. 
To prove (b), we have by (a) and the hypotheses of (b) that sup $\left\|\mu_{n}\right\|$ $<\infty$. Suppose that the conclusion of $(b)$ were false. Then by the same standard sliding hump argument used in (a), we could choose a $\delta>0$, a subsequence $\left(v_{n}\right)$ of the $\mu_{n}$ 's, and a sequence $\left(E_{n}\right)$ of finite disjoint subsets of $A$ such that for all $n,\left|v_{n}\left(E_{n}\right)\right| \geqslant \delta$. Then by Lemma 1.1, there would exist an increasing sequence of indices $\left(n_{i}\right)$ such that for all $i$,

$$
\left|v_{n_{i}}\right|\left(\bigcup_{j \neq i} E_{n_{j}}\right)<\delta / 2 \text {. }
$$

Then putting $E=\bigcup_{j=1}^{\infty} E_{n_{j}}$,

$$
\left|v_{n_{i}}(E)\right|>\delta / 2 \text { for all } i,
$$

contradicting the hypothesis of (b). Q.E.D.

\section{SECTION 2}

This section is deroted to the proof of Lemma 1.1(a).

We first need some notation. For an ordinal number $a, \alpha^{+}$denotes the successor of $\alpha$, and $\bar{\alpha}$ denotes the cardinality of the set of ordinals less than $\alpha$. For a cardinal number $m$, let $o_{m}$ be the least ordinal number $\alpha$ with $\bar{\alpha}=\mathfrak{m}$. For an ordinal number $\eta_{0}$, we shall refer to functions $f$ from the set of ordinals less than $\eta_{0}$ into the ordinal numbers as transfinite sequences, and use the notation $\left(f(\eta) ; \eta<\eta_{0}\right)$ or $(f(\eta))$ when $\eta_{0}$ is understood. Finally, we say that a cardinal number is of type $I$ if it is not the limit of a denumerable sequence of smaller cardinals, and we say it is of type II if it is not of type I.

Our proof proceeds by transfinite induction. Let $m$ be an infinite cardinal number, and assume the lemma has been proved for all infinite cardinal numbers $\mathrm{m}^{\prime}$ with $\mathrm{m}^{\prime}<\mathrm{m}$. (We allow $\mathrm{m}=\mathrm{x}_{0}$ also, in which case this is vacuously true.) Now let $\Gamma$ be given with card $\Gamma=\mathfrak{n t}$, let $S$ be a given m-Stonian compact Hausdorff space, let $\varepsilon>0$, and let $\left\{\mu_{u}: \alpha \in I\right\}$ and $\left\{E_{\alpha}: \alpha \in T\right\}$ be as in the statement of the lemma. Wo now assume (as we may) that $\Gamma$ equals the set of all ordinal numbers less than $o_{\mathfrak{m}}$; " $<$ " denotes the natural order on $\Gamma$.

We shall first prove that there exists a set $\Lambda^{\prime} \subset I^{\prime}$ with $\operatorname{card} \Lambda^{\prime}=\mathrm{n}$, such that

$$
\mu_{\alpha}\left(\bigcup\left\{E_{\beta}: \beta>\alpha, \beta \in \Lambda^{\prime}\right\}\right)<\varepsilon / 2 \text { for all } a \in \Lambda^{\prime} .
$$

We then complete the proof by showing that there exists a set $I^{\prime} \subset \Lambda^{\prime}$ with $\operatorname{card} \Gamma^{\prime}=\mathrm{m}$, such that

$$
\mu_{\alpha}\left(\overline{\bigcup\left\{E_{\beta}: \beta<\alpha, \beta \epsilon \Gamma^{\prime}\right\}}\right)<\varepsilon / 2 \text { for all } \alpha \in \Gamma^{\prime} .
$$

We proceed with the proof of the existence of $\Lambda^{\prime}$. We need the following proposition, which is a variation of a theorem of Tarski (c.f. Theorem 1, page 448 of [25]):

Propositron. There exists a family $\mathscr{F}$ of subsets of $\Gamma$ such that card $\mathscr{F}$ $>\mathrm{m}$; card $\Lambda=\mathrm{m}$ for all $A \epsilon \mathscr{F}$; and if $\Lambda$ and $\Lambda^{\prime}$ are distinct members of $\mathscr{F}$, then there is a $\beta<o_{\mathrm{m}}$ such that $\alpha \in \Lambda \cap \Lambda^{\prime} \Rightarrow \alpha \leqslant \beta$.

We shall use this proposition for now; we give a proof of it at the end of this section, for the sake of completeness.

Choose $\mathscr{F}$ a family of subsets of $\Gamma$, satisfying the conclusion of the proposition. For each $A \epsilon \mathscr{F}$, put

$$
F_{.1}=\bigcap_{\beta \in \Lambda} \overline{\left.\bigcup E_{a}: \alpha \in \Lambda, \alpha \geqslant \beta\right\}} .
$$

Since $S$ is m-Stonian, $F_{A} \cap F_{A^{\prime}}=\emptyset$ for all $A, A^{\prime} \in F$ with $A \neq A^{\prime}$. Now for each $\alpha$, the set of $\Lambda \in F$ such that $\mu_{\alpha}\left(F_{A}\right) \neq 0$ is countable. Thus the set of $\Lambda$ 's in $F$ such that there exists an $\alpha \in \Gamma$ with $\mu_{a}\left(F_{\Lambda}\right) \neq 0$ has cardinality at most $\boldsymbol{\aleph}_{0} \cdot \mathrm{m}=\mathrm{m}<\operatorname{card} \mathscr{F}$. Hence we may choose a $\Lambda \in \mathscr{F}$ with $\mu_{a}\left(F_{1}\right)=0$ for all $a \in \Gamma$.

Fixing $\alpha$, it then follows from the regularity of $\mu_{\alpha}$ if $m$ is of type $I$ (resp. the countable additivity of $\mu_{\alpha}$ if $m$ is of type II) that

$$
\inf _{\beta \in \Lambda} \mu_{\alpha} \overline{\left(\left\{\bigcup E_{\gamma}: \gamma \geqslant \beta, \gamma \in A\right\}\right)}=0 .
$$

Now for each $\beta \in \Lambda$, put

$$
\Lambda_{\beta}=\left\{\alpha \in \Lambda: \alpha<\beta \text { and } \mu_{\alpha}\left(\overline{\left.\bigcup E_{\gamma}: \gamma \geqslant \beta, \gamma \in \Lambda\right\}}\right)<\varepsilon / 4\right\} .
$$

We now define for some $\eta_{0} \leqslant o_{\mathrm{nt}}$, a transfinite sequence $\left\{\alpha(\eta) ; \eta<\eta_{0}\right\}$ of elements of $\Lambda$ as follows: Let $\alpha(0)$ be the least element of $\Lambda$. Let $\eta \leqslant o_{m}$. If $\alpha(\eta)$ has been defined, choose $\beta \in \Lambda$ with $\beta>\alpha(\eta)$ such that

$$
\operatorname{card} \Lambda_{\beta} \cap\{\tau \in A: \tau \geqslant \alpha(\eta)\} \geqslant \overline{\alpha(\eta)} .
$$

(This is possible, since $\overline{\alpha(\eta)}<\mathrm{m}$ and by (1), $A=\bigcup_{\beta \in A} A_{\beta}$ (and by definition, $A_{\beta_{1}} \subset A_{\beta_{2}}$ if $\left.\beta_{1}<\beta_{2}\right)$ ) Then put $\alpha\left(n^{+}\right)=\beta$.

If $\eta$ is a limit ordinal and $\alpha\left(\eta^{\prime}\right)$ has been defined for all $\eta^{\prime}<\eta$, then if $\sup \alpha\left(\eta^{\prime}\right)<o_{\mathrm{m}}$, we choose a $\beta \in \Lambda$ such that $\beta>\alpha\left(\eta^{\prime}\right)$ for all $\eta^{\prime}<\eta$,

and put $\alpha(\eta)=\beta$. (This is possible since card $1=\mathrm{m}$ and $\tau<o_{\mathrm{m}} \Rightarrow \bar{\tau}<\mathrm{m}$.) If $\sup _{\eta^{\prime}<\eta} \alpha\left(\eta^{\prime}\right)=o_{\mathrm{m}}$, we put $\eta_{0}=\eta$ and thus complete the definition of the transfinite sequence $(\alpha(\eta))$. We then have that $(\alpha(\eta))$ is strictly increasing, with $\sup _{\eta<\eta_{0}} \alpha(\eta)=o_{\mathrm{m}}$, and thus $\sup _{\eta<\eta_{0}} \overline{\alpha(\eta)}=\mathrm{m}$. For each $\eta$ with $\eta<\eta_{0}$, put

$$
\Lambda_{\eta}^{*}=\Lambda_{\alpha(\eta+)} \cap\{\tau \epsilon \Lambda: \tau \geqslant \alpha(\eta)\} .
$$


We have that the $A_{\eta}^{*}$ s are pairwise disjoint; indeed $(\alpha(\eta))$ is a strictily increasing sequence and if $\tau \epsilon \Lambda_{\eta}^{*}$, then

$$
\alpha(\eta) \leqslant \tau<\alpha\left(\eta^{+}\right) \text {. }
$$

Thus

$$
\text { card } \bigcup_{\eta<\eta_{0}} A_{\eta}^{*}=\lim _{\eta \rightarrow \eta_{0}} \overline{\alpha(\eta)}=\mathrm{m}
$$

Now let $\eta<\eta_{0}$. Since $\operatorname{card} \Lambda_{\eta}^{*} \leqslant \overline{\alpha\left(\eta^{+}\right)}<m$, by induction hypothesis we may choose a subset $\Lambda_{\eta}^{* *}$ of $\Lambda_{\eta}^{*}$ with $\operatorname{card} \Lambda_{\eta}^{* *}=\operatorname{cord} \Lambda_{\eta}^{*}$ such that

$$
\mu_{\tau}\left(\bigcup\left\{E_{\beta}: \beta \in \Lambda_{\eta}^{* *}, \beta \neq \tau\right\}\right)<\varepsilon / 4 \quad \text { for all } \tau \in \Lambda_{\eta}^{* *}
$$

if $A_{\eta}^{*}$ is infinite; otherwise we let $\Lambda_{\eta}^{* *}$ be a singletion subset of $\Lambda_{\eta}^{*}$.

We now put $\Lambda^{\prime}=\bigcup_{\eta<\eta_{0}} \Lambda_{\eta}^{* *}$. Since card $\Lambda_{\eta}=\overline{\alpha(\eta)}$ for all $\eta<\eta_{0}$, we have that $\operatorname{card} \Lambda^{\prime}=\mathrm{m}$. Now let $\tau \in \Lambda^{\prime}$; so choose $\eta<\eta_{0}$ with $\tau \in \Lambda_{\eta}^{* *}$. Since $\Lambda_{\eta}^{* *} \subset \Lambda_{\alpha(\eta+)}$, we have that

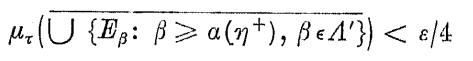

by the definition of $\Lambda_{\alpha\left(\eta^{+}\right)}$. But it follows from (2) that if $\beta \in A^{\prime} \sim A_{\eta}^{* * *}$ and $\beta>\tau$, then $\beta \geqslant \alpha\left(\eta^{+}\right)$. Hence by $(3)$ and the definition of $\Lambda_{a\left(\eta^{+}\right)}$, (*) holds. (If $\mathrm{m}$ is a regular cardinal (i.e. if $o_{\mathfrak{m}}$ is a regular initial number, as defined on p. 403 of [25]), the existence of $\Lambda^{\prime}$ follows easily from (1).)

Now for each $\gamma_{1}, \gamma_{2} \in \Lambda^{\prime}$ with $\gamma_{1}<\gamma_{2}$, set

$$
\Lambda_{\gamma_{1}, \gamma_{2}}=\left\{\alpha \in \Lambda^{\prime}: \mu_{\alpha}\left(\overline{\bigcup\left\{E_{\gamma}: \gamma_{1} \leqslant \gamma<\gamma_{2}, \gamma \in \Lambda^{\prime}\right\}}\right)<\varepsilon / 4\right\} .
$$

We claim that we can choose a $\gamma_{0} \in \Lambda^{\prime}$ such that for all $\beta \in \Lambda^{\prime}$ with $\beta>\gamma_{0}$, card $\Lambda_{\gamma_{0}, \beta}=\mathrm{m}$. Indeed, if this were not true, let $N$ be an integer with $1 / N<\varepsilon / 4$. We could choose $\alpha_{0}$ an arbitrary element of $\Lambda^{\prime}$ and having chosen $\alpha_{n}$ (with $n<N$ ), we could choose $\alpha_{n+1}>\alpha_{n}$ such that card $\Lambda_{a_{n}, \alpha_{n+1}}$ $<$ m. Then

$$
\operatorname{card} \bigcup_{n=0}^{N-1} \Lambda_{a_{n}, a_{n+1}}<\mathrm{m} .
$$

But then we could choose an $\alpha \in \Lambda^{\prime}$ such that $\alpha \notin \Lambda_{a_{n}, \alpha_{n+1}}$ for any $n$ with
$0 \leqslant n \leqslant N-1$. Then

$$
\mu_{a}\left(\bigcup\left\{E_{n}: a_{n} \leqslant \gamma<a_{n+1}\right\}\right) \geqslant \varepsilon / 1 \quad \text { for all } n,
$$

and hence since $S$ is m-Stonian, $\left\|\mu_{a}\right\| \geqslant N(\varepsilon / 4)>1$, contradicting our hypothesis that $\left\|\mu_{a}\right\| \leqslant 1$ for all $\alpha$.

Now choose such a $\gamma_{0}$, and for all $\beta \in \Lambda^{\prime}$ with $\beta \geqslant \gamma_{0}$, put $\Lambda_{\beta}^{\prime}=\left\{\alpha \in \Lambda_{\gamma_{0}, \beta}: \beta<\alpha\right\}$. We thus have that card $\Lambda_{\beta}^{\prime}=\mathrm{m}$, with

$$
\mu_{a}\left(\overline{\left.\bigcup E_{\gamma}: \gamma_{0} \leqslant \gamma<\beta, \gamma \in \Lambda^{\prime}\right\}}\right)<\varepsilon / 4 \quad \text { for all } \alpha \in \Lambda_{\beta}^{\prime} .
$$

The proof now proceeds as in the proof of the existence of $A^{\prime}$. We define for some $\eta_{0} \leqslant o_{\mathrm{m}}$ a transfinite sequence $\left(\gamma(\eta) ; \eta<\eta_{0}\right)$ of elements of $\Lambda^{\prime}$ as follows: $\gamma(0)=\gamma_{0}$. Let $\eta \leqslant o_{\mathrm{nt}}$; if $\gamma(\eta)$ has been defined, let $\gamma\left(\eta^{+}\right)$ be the least $\gamma \in \Lambda_{\gamma^{\prime}(\eta)}^{\prime}$ such that

$$
\operatorname{card}\left\{\tau \in A_{\gamma^{\prime}(\eta)}^{\prime}: \tau<\gamma^{\prime}\right\}=\overline{\gamma(\eta)} \text {. }
$$

Now suppose that $\eta$ is a limit ordinal, and that $a\left(\eta^{\prime}\right)$ has been defined for all $\eta^{\prime}<\eta$. If $\sup _{\eta^{\prime}<\eta} \gamma\left(\eta^{\prime}\right)<o_{\mathrm{m}}$, we may choose a $\tau \in A^{\prime}$ such that $\tau>\gamma\left(\eta^{\prime}\right)$ for all $\eta^{\prime}<\eta$. Then we put $\gamma^{\prime}(\eta)$ equal to the least member of $A_{\tau}^{\prime}$. Continuing in this manner, we will eventually reach an ordinal $\eta_{0} \leqslant o_{\mathfrak{m}}$ such that $\sup \gamma(\eta)=o_{\mathrm{nt}}$, thus completing the definition of $\left(\gamma(\eta) ; \eta<\eta_{0}\right)$. $\eta<\eta_{0}$

Thus, $\gamma(\eta)$ is a strictly increasing sequence of elements of $A^{\prime}$ with $\sup \gamma(\eta)=o_{\mathfrak{m}}$. Now for each $\eta<\eta_{0}$, put $\eta<\eta_{0}$

$$
A_{\eta}^{\prime \prime}=\left\{\tau \epsilon A_{\gamma(\eta)}^{\prime}: \tau<\gamma\left(\eta^{+}\right)\right\}
$$

then if $\beta \epsilon A_{\eta}^{\prime \prime}, \gamma(\eta)<\beta<\gamma\left(\eta^{+}\right)$. Now fix $\eta<\eta_{0}$. Let $\alpha \in A_{\eta}^{\prime \prime}$. Then if $\beta \in \Lambda_{\eta^{\prime}}^{\prime \prime}$ for some $\eta^{\prime} \neq \eta$ and $\beta<\alpha$, then $\eta^{\prime}<\eta$ and consequently $\beta<\gamma(\eta)$. Thus by (4) we obtain that

$$
\begin{aligned}
& \mu_{a}\left(\bigcup\left\{E_{\beta}: \beta<\alpha \text { and } \beta \epsilon A_{\eta^{\prime}}^{\prime \prime} \text { for some } \eta^{\prime} \neq \eta\right\}\right) \\
& \text { for all } \alpha \epsilon A_{\eta}^{\prime \prime} .
\end{aligned}
$$

We have by $(5)$ that $\operatorname{card} A_{\eta}^{\prime \prime}=\overline{\gamma(\eta)}<\mathrm{m}$, hence by induction hypothesis, if $A_{\eta}^{\prime \prime}$ is infinite, we may choose a set $A_{\eta}^{\prime \prime \prime} \subset A_{\eta}^{\prime \prime}$ with card $A_{\eta}^{\prime \prime \prime}$ $=\operatorname{card} A_{\eta}^{\prime \prime}$ such that

$$
\mu_{\alpha} \overline{\left(\bigcup\left\{E_{\beta}: \beta \in A_{\eta}^{\prime \prime \prime}, \beta \neq \alpha\right\}\right.}<\varepsilon / 4 \quad \text { for all } \alpha \in \Lambda_{\eta}^{\prime \prime \prime},
$$

if $A_{\eta}^{\prime \prime}$ is infinite. Again if $\Lambda_{\eta}^{\prime \prime}$ is finite, let $A_{\eta}^{\prime \prime \prime}$ be a singleton subset of $A_{\eta}^{\prime \prime}$. Finally put $\Gamma^{\prime}=\bigcup_{\eta<\eta_{0}} \Lambda_{\eta}^{\prime \prime \prime}$. Then the $\Lambda_{\eta}^{\prime \prime \prime}$ 's are pairwise disjoint (since the $A_{\eta}^{\prime \prime}$ 's are) and by ${ }^{\eta<\eta_{0}}(\tilde{5}), \operatorname{card} \Gamma^{\prime}=\sup _{\eta<\eta_{0}} \overline{\gamma(\eta)}=\mathfrak{m}$. Thus by (6) and (7), $\Gamma^{\prime}$ satisfies (**) and hence the conclusion of Lemma 1.1(a). Q.E.D.

We pass now to the

Proof of the Proposition. We first deal with functions $f: \Gamma \rightarrow \Gamma$. We say that such a function respects the ordering if $f(x)<\omega$ for all $x<\omega$ and $\overline{f(x)} \leqslant \bar{x}$ for all $x$ with $\omega \leqslant x<o_{\mathrm{m}}$. ( $\omega$ denotes the first infinite ordinal, and is identified with the set of finite ordinals.) Evidently $f(x) \equiv x$ is a function respecting the ordering. We denote the graph of $f: \Gamma \rightarrow \Gamma$ by $G_{f}$ (i.e., $G_{f}=\left\{(x, f(x)): x<o_{\mathrm{m}}\right\}$ ). Given $f$ and $g$, we say that $G_{f} \cap G_{g}$ is small, if there is a $\beta<o_{\mathfrak{m}}$ such that for all $x, f(x)=g(x) \Rightarrow x<\beta$. 
We next observe that

$(\Delta) \quad$ if $\mathscr{G}$ is a family of functions respecting the ordering with card $\mathscr{G} \leqslant \mathrm{m}$, then there is a function $g$ respecting the ordering such that $G_{g} \cap G_{f}$ is small for all $f \in \mathscr{G}$.

To see this, let $x \rightarrow f_{x}$ be a surjective correspondence of $I$ into $\mathscr{G}$ (not necessarily one-one). Now fix $y<o_{\mathfrak{m}}$ and let $\beta=\sup _{z \leqslant y} f_{z}(y)$. If $y$ is finite then $\beta$ is finite, and if $y$ is infinite then since $f_{z}$ respects the ordering for all $z, \overline{f_{z}(y)} \leqslant \bar{y}$ for all $z \leqslant y$, and thus $\bar{\beta} \leqslant \bar{y}$. Now we define $g(y)=\beta^{+}$. $g$ then serves as the desired function.

Now by Zorn's Lemma, there exists a non-empty family $\mathscr{G}$ of functions from $\Gamma$ into $T$, maximal with respect to the property: all members of $\mathscr{G}$ respect the ordering and $G_{f} \cap G_{g}$ is small for all $f \neq g$ in $\mathscr{G}$. By $(\Delta)$, we have that $\operatorname{card} \mathscr{G}>\mathrm{m}$.

Finally, we observe that there is a bijective correspondence $p: I \times$ $\times \Gamma \rightarrow \Gamma$ such that $\overline{\varphi(x, y)} \leqslant \max \{\bar{x}, \bar{y}\}$ if either $x$ or $y$ is infinite, and $\varphi(x, y)$ is finite if $x$ and $y$ are both finite. (For each infinite cardinal $\mathfrak{n}<\mathfrak{m}$, let $\varphi_{\mathfrak{n}}$ be a bijective correspondence between $\{(x, y): x, y \in \Gamma$ with $\max \{\bar{x}, \bar{y}\}=\mathfrak{n}\}$ and $\{x \in \Gamma: \bar{x}=\mathfrak{n}\}$. Let $\varphi_{0}$ be a bijective correspondence between $\omega \times(\omega)$ and $\omega$. Then $\varphi=\bigcup_{n<n} \varphi_{n} \cup \varphi_{0}$ is the desired correspondence.)

Then $\mathscr{F}=\left\{\varphi\left(G_{f}\right): f \in \mathscr{G}\right\}$ satisfies the conclusion of the Proposition. Certainly for all $f \in \mathscr{G}$, card $G_{f}=\mathfrak{m}$ and since $\varphi$ is one-one, $\phi\left(G_{f}\right)$ has cardinality $\mathrm{m}$; moreover card $\mathscr{F}=\operatorname{card} \mathscr{G}>\mathrm{m}$. Finally fix $f \neq g$ in $\mathscr{G}$. Then there is a $\beta$ with $0<\beta<o_{\text {m1 }}$ such that $f(x)=g(x) \Rightarrow x<\beta$. Letting $\gamma=\sup \{\varphi(x, f(x)): x<\beta\}, \bar{\gamma} \leqslant \bar{\beta}<m$ if $\beta$ is infinite and $\gamma$ is finite if $\beta$ is finite (by the definition of $\varphi$ and the fact that $f$ respects the ordering). Hence if $z \in \varphi\left(G_{f}\right) \cap \varphi\left(G_{g}\right)$, then $z=\varphi(x, f(x))$ for some $x<\beta$, and thus $z \leqslant \gamma<o_{\mathfrak{m}}$. Q.E.D.

\section{SECTION 3}

In this section we formalize the notion of relatively disjoint families of measures. This notion is of course implicit in much of the preceding work in Banach space theory (c.f. e.g., [7], [9], [10], [16], and [18]). It seems to us that one motivation for crystalizing this concept is the notational ease this provides in its applications.

Throughout this section, $(E, \Sigma)$ shall always denote a set $E$ and a $\sigma$-algebra $\Sigma$ of subsets of $E$.

Definition. Let $(E, \Sigma)$, a family $F=\left\{\mu_{\alpha}: \alpha \in I^{\eta}\right\} \subset \mathrm{ca}(E, \Sigma)$, and $\delta, \varepsilon$ with $0<\varepsilon<\delta$ be given. $F$ is said to be $(\delta, \varepsilon)$-relatively disjoint if $F$ is bounded (i.e. $\sup _{\alpha \in \Gamma}\left\|\mu_{a}\right\|<\infty$ ) and if there exists a family $\left\{\boldsymbol{E}_{a}: \alpha \in \Gamma\right\}$ of disjoint members of $\Sigma$ such that for all $a \in \Gamma$,

$$
\left|\mu_{\alpha}\right|\left(E_{\alpha}\right)>\delta \quad \text { and } \quad \sum_{\beta \neq \alpha}\left|\mu_{\alpha}\right|\left(E_{\beta}\right)<\varepsilon .
$$

$F^{\prime}$ is said to be relatively disjoint if $F$ is $(\partial, \varepsilon)$-relatively disjoint for some $\varepsilon, \delta$ with $0<\varepsilon<\delta$.

If $F$ is a subset of $L^{1}(E, \Sigma, \mu)$ for some measure space $(E, \Sigma, \mu)$, then $F$ is said to be relatively disjoint provided the associated family of measures $\{v: d v=f d \mu$ for some $f \in F\}$ has this property. Finally, we shall refer to denumerably infinite relatively disjoint families as relatively disjoint sequences.

Some of our motivation for explicitly formulating this notion stems from the first result of this section. Variations of it have appeared elsewhere (c.f. [9]) and its proof is essentially known. We first recall the

Definition. Given a set $\Gamma$, let $e_{\alpha}=\chi_{\{a\}}$ for all $\alpha \in I$. We call $\left\{e_{a}: \alpha \in \Gamma\right\}$ the unit-vectors-basis of $c_{0}(\Gamma)$ (resp. of $\left.l^{(}(T)\right)$. Given a Banach space $X$ and a subset $K$ of $X$, we say that $K$ is equivalent to the unit-vectors-basis of $c_{0}(\Gamma)$ (resp. of $l^{1}(T)$ ) provided there exists an isomorphism $T$ from $c_{0}(\Gamma)$ (resp. from $l^{1}(\Gamma)$ ) into $X$ such that $\left\{T e_{a}: \alpha \in \Gamma\right\}=K$.

(The reader should also note that we use "span" to mean "closed linear span".)

Propostition 3.1. Given $(E, \Sigma)$, let $\left\{\mu_{a}: a \in I\right\}$ be a relatively disjoint family in ca $(E, \Sigma)$. Then $\left\{\mu_{a}: \alpha \in \Gamma\right\}$ is equivalent to the unit-vectors-basis of $l^{1}(I)$ and the span of $\left\{\mu_{\alpha}: \alpha \in \Gamma\right\}$ is complemented in ca $(E, \Sigma)$.

Proof. Choose $0<\varepsilon<\delta$ such that the $\mu_{\alpha}^{\prime}$ 's are $(\delta, \varepsilon)$-relatively disjoint, and choose $\left\{E_{a}: a \epsilon \Gamma\right\}$ a family of disjoint members of $\Sigma$ such that (8) holds for all $\alpha \in \Gamma$. Choose for each $\alpha$, a $\Sigma$-measureable function $\varphi_{a}$ supported on $E_{a}$, such that $\int \varphi_{a} d \mu_{a}=\left|\mu_{a}\right|\left(E_{a}\right)$ with $\left\|\varphi_{a}\right\|=1$. Now let $n$ scalars $\lambda_{1}, \ldots, \lambda_{n}$ and $n$ distinct members $\alpha_{1}, \ldots, a_{n}$ of $\Gamma$ be given. Then for each $i$,

$$
\mid \int q_{a_{i}} d\left(\sum_{j} \lambda_{j} \mu_{a_{j}}|| \geqslant\left|\lambda_{i}\right| \delta-\sum_{j \neq i}\left|\lambda_{j}\right|\left|\mu_{a_{j}}\right|\left(E_{a_{i}}\right) .\right.
$$

Hence since the $\varphi_{a_{i}}$ 's disjointly supported,

$$
\begin{aligned}
\| \sum_{j} \lambda_{j} \mu_{a_{j}} \mid & \geqslant\left|\int \sum_{i} \varphi_{a_{i}} d\left(\sum_{j} \lambda_{j} \mu_{u_{j}}\right)\right| \geqslant \delta \sum_{i}\left|\lambda_{i}\right|-\sum_{j}\left|\lambda_{j}\right| \sum_{i \neq j}\left|\mu_{a_{j}}\right|\left(E_{a_{i}}\right) \\
& \geqslant(\delta-\varepsilon) \sum_{i}\left|\lambda_{i}\right| .
\end{aligned}
$$

Since $\sup _{a \in \Gamma}\left|\mu_{a}\right|<\infty$, this proves that $\left\{\mu_{a}: \alpha \in \Gamma\right\}$ is equivalent to the unitvectors-basis of $l^{1}(T)$. 
Now let $W$ be the span of the $\mu_{a}$ 's, $Z$ the span of $\left\{\mu_{a} \mid E_{a}: a \in \Gamma\right\}$, $U: W \rightarrow Z$ the invertible operator defined by $U\left(\Sigma \lambda_{a} \mu_{t a}\right)=\Sigma \lambda_{a} \mu_{a} \mid E_{a}$, and $R$ the projection of ca $(E, \Sigma)$ onto $Z$ defined by

$$
K(\mu)=\sum_{a \in \Gamma}\left(\int \varphi_{a} d \mu\right)\left(\left|\mu_{a}\right|\left(E_{a}\right)\right)^{-1} \mu_{a} \mid E_{a} \quad \text { for all } \mu \in \operatorname{ca}\left(E, \Sigma^{\prime}\right) .
$$

Then if $\mu \in W$, a simple computation shows that $\| U_{\mu-R}-R \mu$ $\leqslant \varepsilon / \delta\|U \mu\|$, which implies that $R \mid W$ is an isomorphism mapping $W$ onto $Z$. Then $Q=(R \mid W)^{-1} R$ is a projection from ca $(E, \Sigma)$ onto W. Q.E.D.

Remark. We know of no subspace of $l^{1}$, isomorphic to $l^{1}$, which is not spanned by a relatively-clisjoint sequence.

Applying Lemma 1.1 to families of countably additive medsures on a discrete set, we obtain easily

Proposition 3.2. Given $(E, \Sigma)$, let $F=\left\{\mu_{a}: a \in I\right\}$ be a bounded infinite subset of $\mathrm{ca}(E, \Sigma)$ such that there exist $a \quad \delta>0$ and a family $\left\{E_{\alpha}: a \in \Gamma\right\}$ of disjoint members of $\Sigma$ with $\left|\mu_{a}\right|\left(E_{\alpha}\right)>\delta$ for all $\alpha \in I$. Then for all $\varepsilon>0$, there exists a $\Gamma^{\prime} \subset \Gamma$ with card $\Gamma^{\prime}=$ card $T$ such that (8) holds for all $\alpha \in \Gamma^{\prime}$ (where the $\beta$ 's of (8) run through $\Gamma^{\prime}$ ). Thus $F^{\prime}$ contains a relatively disjoint family $F^{\prime \prime}$ with card $F=\operatorname{card} F^{\prime}$.

Proof. For each $a \in \Gamma$, define $\tilde{\mu}_{u}$ on all subsets of $I^{\prime}$ by $\tilde{\mu}_{u}(D)$ $=\sum_{\beta \in D}\left|\mu_{\alpha}\right|\left(E_{\beta}\right)$ for all $D \subset \Gamma$. Then $\left\{\tilde{\mu}_{\alpha}: \alpha \epsilon \Gamma\right\}$ and $\left\{\tilde{E}_{a}=\{\alpha\}: \alpha \in \Gamma\right\}$ satisfy the hypotheses of Lemma 1.1, and 3.2 now follows immediately upon applying the conclusion of 1.1 .

We shall now give applications of the above two propositions; later we shall formalize the notion of strongly relatively disjoint families and give applications of the full strength of 1.1 (i.e. of Lemma 1.1.(a)).

Our first application generalizes a theorem of Köthe [10], and yields as a corollary a generalization of Lemma 1.1 of [22]. This result gives the weakest possible conditions that a subset of $l^{1}(T)$ contain a family equivalent to the unit-vectors-basis of $l^{1}(\Gamma)$.

THeOREm 3.3. Let $K$ be a bounded infinite subset of $l^{1}(L)$, such that there is a $\delta>0$ with $\left\|k_{1}-k_{2}\right\| \geqslant \delta$ for all $k_{1} \neq k_{2}, k_{1}, k_{2} \in K$. Then there exists a subset $K^{*}$ of $K$, such that $K^{*}$ is relatively-disjoint and card $K^{*}$ $=$ card $K$. (Consequently by 3.1, letting $11 t=$ card $K^{*}$, the span of $K^{*}$ is isomorphic to $l_{\mathfrak{m}}^{1}$ and complemented in $\left.l^{1}\left(I^{\top}\right)\right)$.

Proof. We first observe that if $A \subset T$ is such that $\operatorname{card} \Lambda<\operatorname{car} \alpha$, then there exists a $k \in K$ and a finite subset $a \subset I \sim \Lambda$, with $\|k \mid a\| \geqslant \delta / 4$. Indeed since $\operatorname{dim} l^{1}(\Lambda)<\operatorname{card} K$, we can choose distinct $k_{1}$ and $k_{2} \in K$ with $\left\|\left(k_{1}-k_{2}\right) \mid \Lambda\right\|<\delta / 8$ (dim is defined immediately following the proof). Thus there is a finite set $\alpha \subset T \sim \Lambda$ with $\left\|\left(k_{1}-k_{2}\right) \mid \alpha\right\|>\frac{6}{3} \delta$, so for one of $i=1$ or $2,\left\|k_{i} \mid \alpha\right\| \geqslant \frac{3}{8} \delta>\delta / 4$. Now, for each finite subset $\alpha$ of $\Gamma$, choose if possible a $k(\alpha) \in K$ such that $\| k_{(\alpha)}\left(\alpha \| \geqslant \delta / 4\right.$. Let $\Gamma_{1}$ be the family of all finite subset $\alpha$ of $\Gamma$ for which such a $k(\alpha)$ exists, and let $F$ be a maximal family of disjoint members of $\Gamma_{\mathrm{I}}$.

$F$ exists by Zorn's Lemma, and our first observation shows that $\operatorname{card} F=\operatorname{card} K$ (since if $\operatorname{card} F<\operatorname{card} K$, then $\operatorname{card} \cup F<\operatorname{card} K$ ). Hence by Proposition 3.2, $K_{1}=\{k(\alpha): \alpha \in F\}$ contains a relatively disjoint family $K^{*}$ with card $K^{*}=\operatorname{card} K_{1}=\operatorname{card} K$. Q.E.D.

Given a Banach space $X$, by $\operatorname{dim} X$ we mean the least cardinal number corresponding to a spanning subset of $X$. It is a well-known and simple consequence of Zorn's Lemma that given $X$ and given $\delta<1$, there exists a subset $K$ of $S_{X}$ with $\operatorname{dim} X=\operatorname{card} K$ and $\left\|k_{1}-k_{2}\right\| \geqslant \delta$ for all $k_{1} \neq k_{2}, k_{1}, k_{2} \in K$. We thus obtain immediately the following result of Köthe:

COROLLARY. Let a set $T$ be given, and let $A$ be a closed subspace of $l^{1}(\Gamma)$. Then there exists a closed subspace $B \subset A$ with $B$ complemented in $l^{1}(T)$ and $B \approx l_{\mathrm{m}}^{1}$ where $\mathrm{m}=\operatorname{dim} A$.

Remark. By the decomposition method of [18] (c.f. also Proposition 1.4 of [22]), it follows that every complemented subspace of $l^{1}(T)$ is isomorphic to $l^{1}(\Lambda)$ for some $\Lambda$. This result is due to Pełczyński for countable $\Gamma$ [18], and to Köthe for general $T$ [10].

Our next result generalizes Lemma 1.1 of [22]; it is another simple consequence of Theorem 3.3. (An application of the result is mentioned in the first remark following 1.5 above.)

Corollary. Let $A \subset X$ be Banach spaces, $\Gamma$ a set, $m$ an infinite cardinal number, and $T: X \rightarrow l^{1}(T)$ an operator be given, satisfying the following: there exists $a \delta>0$ and a bounded subset $K$ of $A$ with $\operatorname{card} K=\mathrm{nt}$ such that $\left\|T k_{1}-T k_{2}\right\| \geqslant \delta$ for all $k_{1}, k_{2} \in K$ with $k_{1} \neq k_{2}$.

Then $A$ contains a subspace $Y$ isomorphic to $l_{\mathrm{m}}^{1}$ and complemented in $X$, such that $T \mid Y$ is an isomorphism.

Proof. Choose $K^{*} \subset K$ with card $K^{*}=m$ and $T K^{*}$ a relatively disjoint family in $l^{1}(T)$ (this is possible by 3.3). Then by 3.1 , and wellknown properties of the unit-vectors-basis of $l_{\mathrm{m}}^{1}$, letting $Y$ be the span of $K^{*}$, there is a projection $P$ from $l^{1}(T)$ onto $T(Y), T \mid Y$ is an isomorphism, and $T(Y)$ is isomorphic to $l_{\mathfrak{n}}^{1}$. Then $Y \approx l_{\mathfrak{m}}^{1}$ and $(T \mid Y)^{-1} P T$ is a projection from $X$ onto $Y$. Q.E.D.

Our next result generalizes Theorem 5 of [2], and yields some rather weak sufficient conditions that a subset of a Banach space contain a subset equivalent to the unit-vectors-basis of $c_{0}(T)$ for some $T$.

THEOREM 3.4. Let a Banach space $B$ and an infinite set $\Gamma$ be given, and let $\left\{b_{a}: \alpha \in \Gamma\right\} \subset B$ and $K>0$ be such that $\left\|b_{\alpha}\right\|=1$ for all $\alpha \in \Gamma$ and

$$
\left\|\sum_{i=1}^{n} \lambda_{i} b_{a_{i}}\right\| \leqslant K \sup _{i}\left|\lambda_{i}\right|
$$


for any positive integer $n$, scalars $\lambda_{1}, \ldots, \lambda_{n}$, and $n$ distinct members $a_{1}, \ldots, \alpha_{n}$ of $\Gamma$.

Then there exists a subset $\Gamma^{\prime}$ of $\Gamma$ with card $\Gamma^{\prime}=\operatorname{carc} \Gamma$, such that $\left\{b_{\alpha}: \alpha \in \Gamma^{\prime}\right\}$ is equivalent to the unit-vectors-basis of $c_{0}\left(I^{\prime \prime}\right)$.

Proof. The argument is similar to that of Proposition 1.2. For each $\alpha \in \Gamma$, choose $b_{\alpha}^{*} \in B^{*}$ with $\left\|b_{a}^{*}\right\|=1=b_{\alpha}^{*}\left(b_{\alpha}\right)$. Now define the scalar-valued function $f_{\alpha}$ on $\Gamma$ by $f_{\alpha}(\beta)=b_{a}^{*}\left(b_{\beta}\right)$ for all $\beta \in \Gamma$. Then $f_{a}(\alpha)=1$, and by $(9)$ $f_{a} \in l(\Gamma)$ with $\left\|f_{a}\right\|_{1} \leqslant K$. Hence by Proposition 3.2, there exists a $\Gamma^{\prime} \subset I^{\gamma}$ with card $\Gamma^{\prime}=$ card $\Gamma$ such that $\left\{f_{\alpha}: \alpha \in \Gamma^{\prime}\right\}$ is $\left(1, \frac{1}{2}\right)$-relatively disjoint. Then $\left\{b_{a}: a \in \Gamma^{\prime}\right\}$ is equivalent to the unit-rectors-basis of $c_{0}\left(I^{\prime \prime}\right)$. Q.F.D.

Remarks. 1. Theorem 3.4 may be reformulated as follows: Let $B$ and $\Gamma$ be as in the statement of 3.4, and let $T: c_{0}(\Gamma) \rightarrow B$ be an operator such that inf $\left\{\left\|T e_{\gamma}\right\|: \gamma \in \Gamma\right\}>0$, where $\left\{e_{\gamma}: \gamma \in \Gamma\right\}$ is the unit-vectors-basis of $c_{0}(\Gamma)$. Then there exists a $\Gamma^{\prime} \subset \Gamma$ with card $\Gamma^{\prime}=\operatorname{card} \Gamma$ such that $T \mid c_{0}\left(\Gamma^{\prime}\right)$ is an isomorphism.

2. It is proved in Theorem 5 of [2] that if $\Gamma$ is countable and $B, \Gamma$, and $\left\{b_{\alpha}: \alpha \in \Gamma\right\}$ satisfy the hypotheses of 3.4, then the span of the $b_{\alpha}$ 's contains a subspace isomorphic to $c_{0}$. Thus 3.4 contains some new information for countable $\Gamma$ also.

The following result is an application of 3.5 ; it is motivated by the theorem stated after Corollary 1.7 above. (The notation $A \perp B$ is defined preceding 1.7.)

Proposimion 3.5. Let $\Gamma$ be an infinite set and let $A$ be a closed subspace of $l^{\infty}(\Gamma)$ such that there is no $B \subset l^{\infty}(\Gamma)$ with $B \approx c_{0}(\Gamma)$ and $A \perp B$. Then $l^{\infty}(\Gamma)$ is a continuous linear image of $A$. If card $\Gamma$ is not a limit of a sequence of smaller cardinals, then $l^{\infty}(\Gamma)$ is isometric to a subspace of $A$.

We do not know if the hypotheses of 3.4 imply that $A$ contains an isomorph of $l^{\infty}(\Gamma)$, with no restriction on card $\Gamma$. (It is proved in [13] that this holds if $\Gamma$ is countable.)

Pro of. Let $\Lambda$ be a set with card $\Lambda=\operatorname{card} \Gamma$ and let $\left\{\Gamma_{a}: \alpha \in \Lambda\right\}$ be a family of disjoint subsets of $\Gamma$ with $\operatorname{card} \Gamma_{\alpha}=\operatorname{card} \Gamma$ for all $\alpha$. Let $\varepsilon$ be fixed with $0<\varepsilon \leqslant \frac{1}{2}$ and let $B_{\varepsilon}$ be the set of all $a \epsilon \Lambda$ such that there exists a $\varphi_{a} \epsilon l^{\infty}\left(\Gamma_{a}\right)$ satisfying

$$
\left\|\varphi_{a}\right\|=1 \text { and }\left\|\varphi_{a}-a\right\| \geqslant \varepsilon \quad \text { for all } a \in A
$$

Then card $B_{\varepsilon}<\operatorname{card} \Gamma$. Indeed, for each $\alpha \in B_{\varepsilon}$, choose $\varphi_{a} \in l^{\infty}\left(I_{a}\right)$ satisfying $(*)$. Then let $\pi: l^{\infty}(\Gamma) \rightarrow l^{\infty}\left(I^{\prime}\right) / \boldsymbol{A}$ be the quotient map. Now suppose that $\operatorname{card} B_{\varepsilon}=\operatorname{card} \Gamma$. Then $\left\{\varphi_{\alpha}: \alpha \in B_{\varepsilon}\right\}$ is isometrically-equivalent to the unit-vectors-basis of $c_{0}(T)$, and by $(*), \inf \left\{\left\|\pi \varphi_{\alpha}\right\|: \alpha \in B_{\varepsilon}\right\} \geqslant \varepsilon>0$. Hence by Theorem 3.4, there exists a subset $\Gamma^{\prime}$ of $B_{s}$ with card $\Gamma^{\prime}=\operatorname{card} T^{\top}$ such that letting $B$ be the span of $\left\{\varphi_{a}: \alpha \in \Gamma^{\prime}\right\}, \pi \mid B$ is an isomorphism.
Then $B \perp A$ and of course $B$ is isometric to $c_{0}(\Gamma)$, which contradicts our hypotheses.

Thus since card $B_{1 / 2}<\operatorname{card} \Gamma$, there exists an $\alpha \epsilon \Lambda$ such that $\alpha \notin B_{1 / 2}$. Then letting $P$ be the canonical projection of $l^{\infty}(\Gamma)$ onto $l^{\infty}\left(\Gamma_{\alpha}\right), P(A)$ $=l^{\infty}\left(\Gamma_{\alpha}\right)$. For $P$ has norm one, and given $\varphi \epsilon l^{\infty}\left(\Gamma_{a}\right)$ with $\|\varphi\|=1$, there exists an $a \in A$ with $\|a-q\|<\frac{1}{2}$, whence $\|P a-\varphi\|<\frac{1}{2}$. The completeness of $A$ and a standard iteration argument then show that there exists an $a_{1} \in A$ with $P a_{1}=q$. Hence $l^{\infty}(\Gamma)$ is a continuous linear image of $A$.

Finally, if $\operatorname{card} \Gamma$ is not the limit of a sequence of smaller cardinals, $\operatorname{card} \bigcup_{n=2}^{\infty} B_{1 / n}<\operatorname{card} \Gamma$, whence there exists an $\alpha \in \Lambda$ with $\alpha \notin \bigcup_{n=2}^{\infty} B_{1 / n}$. Since $A$ is closed, this implies that $l^{\infty}\left(\Gamma_{a}\right) \subset A$, and of course $l^{\infty}\left(\Gamma_{a}\right)$ is isometric to $l^{\infty}(T)$. Q.E.D.

Remark. Let $\Gamma$ be an infinite set and $A$ be a Banach space. It is an easy consequence of known results that the following three assertions are equivalent:

1. There exists an operator $\Gamma: A \rightarrow l^{\infty}(\Gamma)$ with dense range.

2. A contains a subspace isomorphic to $l_{2}^{1} \mathfrak{m}$ where $\mathrm{m}=\operatorname{card} \Gamma$.

3. There exists a surjective operator $T: A \rightarrow l^{\infty}(T)$.

Indeed, it follows easily from a result of Hewitt [8], that $l_{2}^{1} \mathrm{~m}$ is isometric to a subspace of $l^{\infty}(\Gamma)$, where card $\Gamma=\mathrm{m}$ (c.f. the proof of $(d) \Rightarrow(f)$ of Theorem 5.1 of [22]). But then by a result of Pelczyriski (Lemma 4.2 of $[16]$ ), (1) $\Rightarrow(2)$ since $2^{\mathfrak{m}}$ is not a limit of a sequence of smaller cardinals (c.f. the Remark immediately following 4.2 of [16]). Since $\operatorname{dim} l^{\infty}(I)=2^{\mathrm{m}}$, it follows easily that $l^{\infty}(T)$ is isometric to a quotient space of $l_{2}^{1} \mathfrak{m}$, and hence since $l^{\infty}(\Gamma)$ is injective, it is a continuous linear image of any space containing $l_{2}^{1} \mathrm{~m}$, whence $(2) \Rightarrow(3)$.

We pass finally to a formalization of the notion underlying the proof of Proposition 1.2.

Definition. Given a compact Hausdorff space $S$ and a bounded set $F=\left\{\mu_{a}: \alpha \in \Gamma\right\} \subset M(S)=C(S)^{*}$, we say that $F$ is strongly relatively disjoint if there exist a family $\left\{E_{a}: \alpha \in \Gamma\right\}$ of disjoint open sets and $\varepsilon, \delta>0$ with $\varepsilon<\delta$, such that for all $\alpha \in \Gamma$,

$$
\left|\mu_{a}\right|\left(E_{\alpha}\right)>\delta \quad \text { and } \quad\left|\mu_{a}\right|\left(\bigcup_{\beta \neq a} E_{\beta}\right)<\varepsilon .
$$

We may view our argument for 1.2 as an application of strongly relatively disjoint families as follows: Let $B, \Gamma$, and $T$ satisfy the hypotheses of 1.2. Identifying $l^{\infty}(\Gamma)$ with $C(\beta \Gamma)$, our first part of the proof of 1.2 applies Lemma 1.1 to show that $T^{*}\left(S_{B^{*}}\right)$ contains a strongly relatively disjoint family $\Gamma^{\prime}$ with $\operatorname{card} \Gamma^{\prime}=\operatorname{card} \Gamma$. A simple modification of the proof of 1.2 then shows 
Proposition 3.6. Let $\mathrm{m} \geqslant \mathbf{\aleph}_{0}$. Let $\mathrm{S}$ be an m-Stonian compact Hausdorff space, $B$ a Banach space, and $T: C(S) \rightarrow B$ an operator such that there exists a $\Gamma \subset S_{B^{*}}$ with card $\Gamma \leqslant \mathrm{m}$ such that $T^{*}(\Gamma)$ is a strongly relatively disjoint family. Then there is a subspace $A$ of $O(S)$ isometric to $l^{\infty}(T)$ such that $T \mid A$ is an isomorphism.

Proof. For each $\gamma \in \Gamma$, we define $\mu_{\gamma} \in M(S)$ by $\int p d \mu_{\gamma^{\prime}}=\gamma\left(T p^{\prime}\right)$ for all $\phi \in C(S)$. Our hypotheses imply that we may choose $0<\varepsilon<\delta$ and $\left\{E_{\alpha}: \alpha \in T\right\}$ a family of disjoint clopen subsets of $S$ satisfying (10) for all $\alpha \in \Gamma$.

Now choose $\delta^{\prime}$ with $\varepsilon<\delta^{\prime}<\delta$, and for each $\alpha \epsilon I$, choose $\varphi^{\prime}$ eontinuous on $S$, supported on $E_{a}$, of sup norm one, and satisfying

$$
\int \varphi_{\alpha} d \mu_{a} \geqslant \delta^{\prime}
$$

Now put $F=\bigcup_{a \in \Gamma} E_{\alpha}$ and let $A$ denote the set of all $f \in O(S)$ which are supported on $F$ and such that for all $\alpha \epsilon \Gamma, f \mid E_{\alpha}=c_{\alpha} \varphi_{a}$ for some constiant $c_{\alpha}$. Since $S$ is m-Stonian, $F$ is open and every bounded continuous function on $\bigcup_{a} E_{a}$ extends to a continuous function supported on $F$ (c.f. Theorem 14.25, page 208 of [6]). Thus $A$ is isometric to $l^{\infty}(T)$. But fixing $f \in A$ and $\alpha \in \Gamma$,

$$
|\alpha(T f)|=\left|\int_{E_{\alpha}} f d \mu_{a}+\int_{\substack{\cup E_{\beta} \\ \beta \neq \alpha}} f d \mu_{\alpha}\right| \geqslant\left\|f \mid E_{\alpha}\right\|_{\infty} \delta^{\prime}-\|f\|_{\infty} \varepsilon
$$

by (10) and (11). Hence $\|T f\| \geqslant\left(\delta^{\prime}-\varepsilon\right)\|f\|_{\infty}$, proving that $T \mid A$ is an isomorphism. Q.E.D.

Proposition 3.6 permits us to give a different proof of a somewhat stronger result than Corollary 1.4. We first recall the following

THEOREM of GRoTHENDIECK (Théorème 2, page 146 of [7]). Let $S$ be a compact Hausdorff space and let $K$ be a bounded non-weakly conditionally compact subset of $M(S)$. Then there exists a $\delta>0$, a sequence $\left(\mu_{n}\right)$ of members of $K$, and a sequence $\left(E_{n}\right)$ of disjoint open subsets of $S$ such that for all $n$, $\left|\mu_{n}\right|\left(E_{n}\right)>\delta$.

Now 1.1, the argument of the preceding proposition, and the Therem of Grothendieck yield

THEOREM 3.7. Let $S$ be a compact Hausdorff space, $B$ a Banach space, and $T: C(S) \rightarrow B$ a non-weakly compact operator. Then there exists a subspace $A$ of $C(S)$ isometric to $c_{0}$ such that $T \mid A$ is an isomorphism. Moreover if $S$ is $\sigma$-Stonian, then $A$ may be chosen isometric to $7^{\infty}$ and such $T \mid A$ is an isomorphism. Finally, if $S$ is an $F^{-}$-space, then $l^{\infty}$ is a continuous linear image of $B$.

Remark. The first assertion is due to Pelczyński (Theorem 1 of [1.7]).
Proof. It follows immediately from the Theorem of Grothendieck and Proposition 1.2 that there exist a countable infinite $\Gamma \subset S_{B^{*}}$, a family $\left\{E_{\alpha}: \alpha \in \Gamma\right\}$ of disjoint open subsets of $S$, and $0<\varepsilon<\delta$ such that defining the $\mu_{a}$ 's as in the proof of $3.6,(8)$ holds for all $\alpha \epsilon \Gamma$. We then choose $\delta^{\prime}$ and the functions $\varphi_{\gamma}$ exactly as in the proof of 3.6. Then letting $A$ be the closed linear span of the $\varphi_{\gamma}$ 's we have that $A$ is isometric to $c_{0}$ and $T \mid A$ is an isomorphism.

If $S$ is in addition $\sigma$-Stonian, then the $E_{a}$ 's may be chosen to be clopen, whence by Lemma $1.1(\mathrm{a}), T^{*}\left(S_{B^{*}}\right)$ contains a strongly relatively disjoint sequence. Hence by Proposition 3.6, there exists an $A \subset C(S)$ isometric to $l^{\infty}$ such that $T \mid A$ is an isomorphism.

Finally if $S$ is an $F$-space, it follows since $\Gamma$ is countable that there is a closed Stonian subset $K$ of $S$ such that $\left|\mu_{\gamma}\right|(\sim K)=0$ for all $\gamma \in \Gamma$. (Let $K=\overline{\bigcup_{\gamma \in \Gamma} \operatorname{Supp} \mu_{\gamma}}$; then $K$ is a closed subset of an $F$-space and hence an $F$-space, and $K$ satisfies the C.C.C. Hence $K$ is Stonian by the Proposition preceding 1.6 above.) It then follows as in the proof of 3.6 that there exists a subspace $A$ of $C(K)$, isometric to $l^{\infty}$, such that

$$
\sup _{\alpha}\left|\int f d \mu_{\alpha}\right| \geqslant\left(\delta^{\prime}-\varepsilon\right)\|f\|_{\infty} \quad \text { for all } f \in A \text {. }
$$

Then put $A_{1}=\left\{f_{\epsilon} C(S): f \mid K \in A\right\}$, put $B_{1}=\{b \epsilon B: \gamma(b)=0$ for all $\gamma \in T\}$, and let $\tau: B \rightarrow B / B_{1}$ be the quotient map. We then have by (12) that for all $f \in A_{1}$,

$$
\left\|f \left|K\left\|_{\infty} \leqslant\left(\delta^{\prime}-\varepsilon\right)^{-1} \sup _{\gamma \in \Gamma}|\gamma(T f)| \leqslant\left(\delta^{\prime}-\varepsilon\right)^{-1}\right\| \tau T f \| .\right.\right.
$$

Then defining $\varphi: \tau T\left(A_{1}\right) \rightarrow A$ by $\varphi(\tau T f)=f \mid K$ for all $f \epsilon A_{1}$, we have that $\varphi$ is a well-defined surjective linear map with $\|\varphi\| \leqslant\left(\delta^{\prime}-\varepsilon\right)^{-1}$. Since $l^{\infty}$ is injective there exists an operator $\tilde{\varphi}: B / B_{1} \rightarrow A$ extending $\varphi$, whence $\tilde{\varphi} \tau$ maps $B$ onto an isomorph of $l^{\infty}$. Q.E.D.

Remarks. 1. As we noted following 1.4, using a result in [12] and assuming the continuum hypothesis, the second assertion of 3.7 holds if we assume there that $S$ is an $F$-space. Without assuming the continuum hypothesis, however, we do not know if for every $F$-space $S, C(S)$ contains an isomorph of $l^{\infty}$.

2. An immediate consequence of 3.7 is that if the Banach space $X$ is a non-reflexive continuous linear image of $C(S)$ for some $F$-space $S$, then $l^{\infty}$ is a continuous linear image of $X$ (Theorem 6 of [21]). This result has application to quasi-complementation problems (c.f. [23] and Theorem 9 of [21]).

We have used the results of the present paper in the above argument. Actually, the proofs of 3.6 and 3.7 are already implicit in the proof of Theorem 2 of [21], which uses Lemma 1 of [21]. In reality, however, Studia Mathematica xxxvir. 
this argument may be given by using results established prior to Lemma 1 of [21] to prove the following

Propostition. Let $S$ be a compact Hausdorff space and let $K$ be a bounded symmetric $(x \in K \Rightarrow-x \epsilon K)$ convex subset of $M(S)$ such that $\bar{K}$ is not weakly compact. Then $K$ contains a strongly relatively disjoint sequence.

Proof. There exists a $K_{1} \subset K$ such that $K_{1}$ is a convex symmetric subset of $L^{1}(\mu)$ for some finite measure $\mu$, with $\bar{K}_{1}$ non-weakly compact, Then by the proofs of two results of Kadec and Pełczyniski (Theorems 2 and 6 of [9]), there exist a sequence $\left(\mu_{n}\right)$ of elementis of $K, a \delta>0$, and a sequence $\left(E_{n}\right)$ of disjoint Borel sets such that

$$
\delta=\lim \left\|\mu_{n}\right\|=\lim \left|\mu_{n}\right|\left(E_{n}\right) .
$$

Then by a variation of the Theorem of Grothendieck (c.f. Lemma 1 of [15]) there exist a subsequence $\left(v_{n}\right)$ of the $\mu_{n}$ 's and a sequence $\left(O_{n}\right)$ of disjoint open sets such that $\left|\nu_{n}\right|\left(O_{n}\right)>\frac{2}{3} \delta$ for all $n$. Hence automatically $\left|v_{n}\right|\left(\bar{\cup}_{j \neq n} O_{j}\right)<\frac{1}{2} \delta$ for all $n$ sufficiently large, i.e., $\left\{v_{n}: n>N\right\}$ is strongly relatively disjoint, for some $N$.

Remark. It is easily seen that neither the assumption of convexity nor the assumption of symmetry may be omitted in general. Indeed, let $K_{1}$ be the convex hull of $\left\{\delta_{1 / n}-\delta_{0}: n=1,2, \ldots\right\}$ in $M[0,1]$ $=C[0,1]^{*}$ and let $K_{2}=\left\{ \pm\left(\delta_{1 / n}-\delta_{0}\right): n=1,2, \ldots\right\}$. $\left(\delta_{x}\right.$ denotes the measure of norm one assigning mass one to the point $x$ ). Then for $i=1$ and $2, K_{i}$ is not weakly sequentially compact yet contains no strongly relatively disjoint sequence; $K_{1}$ is convex, $K_{2}$ is symmetric.

As our last result, we restate Lemma 1 of [21] in terms of the concepts introduced in this section.

THEOREM. Let $K$ be a bounded non-wealty conditionally compact subset of $B$. Then if $B=M(S)$ for some compact $F$-space $S, K$ contains a strongly relatively disjoint sequence. If $B=\mathrm{ca}(E, \Sigma)$ for some $(E, \Sigma)$, $K$ contains a relatively disjoint sequence. Finally if $B=M(S)$ for some arbitrary compact Hausdorff space $S$, then $K$ contains a relatively disjoin sequenoe $\left(\mu_{\alpha}\right)$ such that the disjoint sets $E_{\alpha}$ satisfying (8) may be chosen open for all $\alpha$.

This theorem follows immediately from the proof of Lemma 1 of [21] (c.f. the paragraph following Theorem 6 of [21]). In the present work, this follows immediately from 1.1, the fact that $F$-spaces satisfying the C.C.C., are Stonian, and the Theorem of Grothendieck (or its simpler analogue for the case when $B=\operatorname{ca}(E, \Sigma)$ (c.f. Theorem 4, page 305 of $[5]))$.

From the Theorem and Proposition 3.1, we obtain immediately the result of Kadec and Pełczyński [9] that every non-reflexive subspace of $L^{1}$ contains a complemented subspace isomorphic to $l^{1}$. We also obtain the result of Grothendieck [7]: If $\left(\lambda_{n}\right)$ is a bounded sequence in $M(S)$ $=O(S)^{*}$ and if $S$ is $\sigma$-Stonian (resp. $S$ is arbitrary), then if $\left(\lambda_{n}\left(F^{\prime}\right)\right)$ is a convergent sequence of scalars for every clopen set $F$ (resp. for every open set $F$ ) then $\left(\lambda_{n}\right)$ is weakly convergent (c.f. the final argument in Section 1).

This result of Grothendieck in turn implies the Lemma of Phillips [20] from which all these results stem.

\section{References}

[1] D. Amir, Projections onto continuous function spaces, Proc. Amer. Math. Soc. 15 (1964), pp. 396-402.

[2] C. Bessaga and A. Pełczyński, On bases and unconditional convergence of series in Banach spaces, Studia Math. 17 (1958), pp. 151-164.

[3] M. M. D ay, Normed Linear Spaces, Berlin 1958.

[4] J. Dieudonné, Sur la convergence des suites de mesures de Radon, Anais. Acad. Brasil. ci. 23 (1951), pp. 21-38, pp. 277-282.

[5] N. D unford and J. T. Schwartz, Linear Operators I, New York 1958.

[6] L. Gillman and M. Jerison, Rings of Continuous Functions, Princeton, N. J., 1960.

[7] A. Grothendieck, Sur les applications lineaires faiblement compactes d'espaces $d u$ type $O(K)$, Can. J. Math. 5 (1953), pp. 129-173.

[8] E. Hewitt, A remark on density characters, Bull. Amer. Math. Soc. 52 (1946), pp. 641-643.

[9] M. I. Kadec and A. Pełczyński, Bases, lacunary sequences, and complemented subspaces in the spaces $L_{p}$, Studia Math. 21 (1962), pp. 161-176.

[10] G. Köthe, Hebbare Lokalkonvexe Raüme, Math. Ann. 165 (1966), pp. 181-195.

[11] J. Lindenstrauss, On complemented subspaces of m, Israel J. Math. 5 (1967), pp. 153-156.

[12] - On the extension of operators with range in a $C(K)$ space, Proc. Amer. Math. Soc. I5 (1964), pp. 218-225.

[13] - and H. P. Rosenthal, Automorphisms in $c_{0}, l_{1}$, and $m$, Israel J. Math. 7 (1969), pp. 221-239.

[14] M. Nakamura and S. Kakutani, Banach limits and the Cech compactification of a countable discrete set, Proc. Imp. Acad. Japan 19 (1943), pp. 224-229.

[15] A. Pełczyński, Banach spaces on which every unconditionally converging operator is weakly compact, Bull. Acad. Pol. Sci., Série Math., Astr. et Phys. 10 (1962), pp. 641-648.

[16] - On Banach spaces containing $L_{1}(\mu)$, Studia Math. 30 (1968), pp. 231-246.

[17] - On strictly singular and strictly cosingular operators I, Bull. Acad. Pol. Sci., Série Math., Astr. et Phys. 13 (1965), pp. 31-36.

[18] - Projections in certain Banach spaces, Studia Math. 19 (1960), pp. 209-228.

[19] - and V. N. Sudakov, Remark on non-complemented subspaces of the space $m(s)$, Coll. Math. 9 (1962), pp. 85-88.

[20] R. S. Phillips, On linear transformations, Trans. Amer. Math. Soc. 48 (1940), pp. 516-541.

[21] H. P. Rosenthal, On complemented and quasi-complemented subspaces of quotients of $O(S)$ for Stonian S, Proc. Nat. Acad. Sci. U. S. 60 (1968), pp. 1165-1169. 
[22] H. P. Rosenthal, On injective Banach spaces and the spaces $L^{\infty}(\mu)$ for fute measures $\mu$, Acta Math. 124 (1970), pp. 205-247.

[23] - On quasi-complemented subspaces of Banach spaces, Proc. Nat. Acad. Sci. U. S. 59 (1968), pp. 361-364.

[24] G. L. Seever, Measures on F spaces, Trans. Amer. Math. Soc. 133 (1968), pp. 267-280.

[25] W. Sierpiński, Cardinal and Ordinal Numbers, Warszawa 1965.

[26] R. Whitley, Projecting $m$ onto $c_{0}$, Amer. Math. Monthly 73 (1966), pp. 285-286.

Reçu par la Rédaction le 26. 9. 1969

\section{Minimal sublinear functionals *}

by

S. SIMONS (Santa Barbara, Calif.)

\section{O. INTROD UCTION}

In Section 1 we consider a class $\mathscr{P}$ of sublinear functionals on a real linear space $E$ and show that $\mathscr{P}$ contains elements minimal with respect to the pointwise ordering on $R^{E}$. The general existence theorem is Theorem 15 and involves the definition of a "boundary" for $\mathscr{P}$ in Notation 13.

In Section 2 we give conditions for an element of $\mathscr{P}$ to dominate a unique minimal element of $\mathscr{P}$.

In Section 3 we give a Shilov theorem for sublinear functionals on $E$.

Under certain conditions (Theorem 12, Notation 23 and Lemma $27(b))$ the minimal elements of $\mathscr{P}$ coincide with the linear elements of $\mathscr{P}$. In Section 6 we deduce various forms of the Hahn-Banach theorem and generalizations of results of Kelley and Sikorski (see Remark 29).

In Section 7 we deduce, with a number of improvements over the known results, Shilov theorems and conditions for the existence and uniqueness of balayages defined by a cone in $\mathscr{C}(X)$ ( $X$ compact Hausdorff) (see Remark 32). There is also a short discussion of the Choquet boundary of a subspace of $\mathscr{C}(X)$ (see Remark 35).

In Section 8 we suppose that $X$ is a compact convex set in a Hausdorff locally convex space and deduce, with a number of improvements, results of Milman, Bauer and Choquet-Meyer (see Remark 38) as well as the Choquet-Bishop-deLeeuw theorem.

We use mainly linear space techniques - the only places where any measure theory is mentioned are Theorem $30(\mathrm{~g})$, Theorem $33(\mathrm{a})$ and Theorem $36(\mathrm{e})$. In Section 9 we apply our results to a "non- $\mathscr{C}(X)$ " situation, replacing $\mathscr{C}(X)$ by the set of continuous affine functions on a compact convex set (in a Hausdorff locally convex space).

In Section 10 we make some further observations about the uniqueness problem.

* This research was supported in part by NSF grant GP 8394. 